typical attack the patient first suffers a febrile, catarrhal illness for 10 days to a fortnight and then passes into the stage of spasmodic coughing. Because they do not expect whooping-cough in the adult, both patient and doctor may regard this stage for some time as an unusually severe form of bronchitis, but it may become so distressing that eventually they suspect the true diagnosis. The whoop is not usually so pronounced in the adult as in the young child, and the adult often learns to control it. Vomiting, too, is less common in the adult, but when cough, whoop, and vomit all occur there can be little doubt about the nature of the illness. In atypical attacks the adult patient suffers simply from the early febrile catarrhal illness or from a respiratory illness indistinguishable from acute bronchitis. In such cases the true diagnosis is likely to be missed, even when there has been close contact with children with whooping-cough, unless the doctor is aware that the disease can occur at any age and with symptoms very unlike the classical cough, whoop, and vomit of young children.

The difficulty of the diagnosis in adults is well illustrated by the report of two outbreaks among hospital staff of a paediatric unit in Denver, U.S.A. 6 In one, a house officer developed a mild respiratory illness after contact with a child with whooping-cough. He infected his wife, a ward clerk, and two children whom he saw as outpatients. A nurse caught the infection from one of these children, and she infected her husband and two other nurses. In the second outbreak a graduate student caught whooping-cough from children she attended in their homes. She infected another student, who in turn infected a nurse and a third student. Two of the adult patients had severe cough and loss of weight, and one of them had paroxysmal cough on effort for more than three months. Except for these severe attacks the other milder respiratory illnesses and the chain of infection from adults to children might have gone unrecognized, and this is epidemiologically important because it is in the early, non-paroxysmal, catarrhal stage that whooping-cough is most infectious. As it was, diagnosis was confirmed by culture and fluorescent antibody examination of nasopharyngeal swabs, and titration of sera for rise in pertussis agglutination titres.

Similar incidents have been reported, 5 but they are probably not common. They may occur when perhaps the strain of Bordetella pertussis is unusually invasive or the level of adult immunity unusually low, convenient assumptions for which there is no epidemiological evidence. To advocate serum antibody titration in all who have to look after children with whooping-cough and to immunize those who appear to lack immunity might be getting things out of proportion. But one should be aware of the risk. Doctors, nurses, and other attendants who develop coughs and colds when looking after children with whooping-cough should be investigated bacteriologically and isolated till results are known. In the early catarrhal stage antibiotics such as the tetracyclines or erythromycin may help to eradicate the organism and modify the clinical attack. In the paroxysmal stage antibiotics have no effect on the illness, but by then the patient is rapidly becoming non-infectious.

\footnotetext{
1 Mannerstedt, G., fournal of Pediatrics, 1934, 5, 596.

Morse, S. I., Annals of Internal Medicine, 1968, 68, 953.

Christie, A. B., Infectious Diseases: Epidemiology and Clinical Practice, p. 699, 1969. Edinburgh: Livingstone.

- Horner, F. A., New England Fournal of Medicine, 1962, 266, 470.

- Lambert, H. J., Public Health Reports, 1965, 80, 365.

- Kurt, T. L., Yeager, A. S., Guenette, S., and Dunlop, S., fournal of the American Medical Association, 1972, 221, 264.
}

\section{Some Problems of Acute Osteomyelitis}

Antibiotics have had an increasingly important role in the treatment of acute osteomyelitis in children. Indeed, without them the control of the infection would be almost impossible. But over the years resistance to the original antibiotics has developed, and in 1970 N. J. Blockey and J. T. Watson ${ }^{1}$ reported on it in a review of 113 children who were treated for acute osteomyelitis between 1961 and 1968. Organisms were cultured from blood and pus in 79 patients, and in 68 of them (86\%) Staphylococcus aureus was found. In 1946 $14.1 \%$ of staphylococci were resistant to benzylpenicillin, and by 1970 this had increased to $93 \% .^{2}$ The resistance of Staph. aureus was almost exclusively confined to penicillin, streptomycin, and tetracycline. Blockey and T. A. McAllister ${ }^{2}$ showed that, though resistance to penicillin has increased, staphylococci resistant to penicillin were always sensitive to cloxacillin, though they consider that Staph. aureus resistant to cloxacillin and methicillin will be found in acute osteomyelitis of children. They concluded that careful choice of drugs should prevent the development of resistance, provide a broad spectrum of activity, and show synergy, and suggested fusidic acid and erythromycin. They decided that from January 1969 children with acute osteomyelitis should initially receive fusidic acid and benzylpenicillin, and, if there was evidence of resistance to penicillin, erythromycin should be substituted. From January 1969 to May 1970 this antibiotic combination was given to 38 patients. It was found that fusidic acid gave slightly better results with erythromycin or benzylpenicillin ( $10.5 \%$ failures) than cloxacillin and benzylpenicillin in a previous series (14.5\% failures). In addition to antibiotic therapy rest by immobilization of the affected limb is important, though it is apt to be overlooked. The Thomas splint is very suitable for the lower limb, for it allows the affected area to remain exposed for an examination. For the upper limb plaster-of-Paris slabs should be used.

In arriving at a diagnosis careful palpation is important, and if pain is severe a general anaesthetic may be necessary. The bulky muscles round the thigh and knee may make it difficult to detect an abscess, and in some regions such as the hip it may be impossible to detect it by palpation even with an anaesthetic. In these circumstances the area should be explored with an aspirating needle and syringe, and aspiration should include the hip joint. It is also worth bearing in mind that osteomyelitis may involve the scapula and pelvic bones.

Surgery should not be undertaken unless there is clear evidence of a superiosteal abscess, and even then drainage by incision or aspiration is not proved to be essential in the treatment of osteomyelitis. Drilling of the metaphysial region has in the past been advocated, but most surgeons now regard it as unnecessary at any stage, and it may even be harmful.

Successful primary treatment was defined by Blockey and Watson ${ }^{1}$ as cure of the symptoms and signs within four weeks of the first admission to hospital, with no complication attributed to local bone disease appearing thereafter. In all 21 cases in which treatment failed by these criteria the bone infection disclosed itself within seven months of leaving hospital. It would seem therefore that the correct treatment for this condition has not yet been decided. We do not know if the use of a powerful antibiotic is sufficient without drain- 
age of an abscess. Nor do we know if quiescence of infection for long periods after the treatment has been concluded indicates permanent healing, for the infection may recur many years after the initial treatment has been completed.

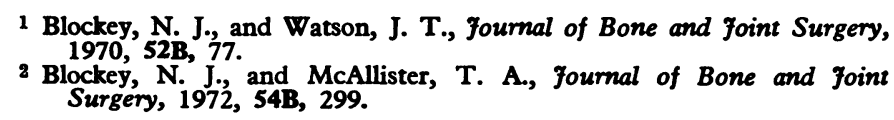

\section{Seventy Years On}

Sir Paul Chambers's proposals for reorganizing the B.M.A.' have been debated thoroughly by the Association's many committees during the past six months. The Council has given its views in two reports ${ }^{2-3}$ and these, along with the Chambers Report, have been discussed in divisions and branches as well as by local medical committees. The final decision on how-perhaps even whether-the profession's main representative organization should reshape itself now lies with the Representative Body when it meets on 15 November, one week after a special conference of L.M.C. representatives has debated the General Medical Services Committee's report on Sir Paul's proposals.

In 1901 the B.M.A., then only 18,000 strong, was studying a provisional report ${ }^{3}$ proposing reforms of its constitution. "Arrangements were made in many areas for its discussion .. . and a considerable number of meetings took place throughout the United Kingdom. Yet the attendances were in most places meagre . . . the total members present at all the meetings was only about 2,000 .. Probably it must always be so in matters of medical politics." 4 Things have changed little 70 years on. Even when Sir Paul has taken the trouble to visit division or branch meetings to explain his report the audiences seem to have risen little above the customary 5 to $10 \%$ of the membership. Are doctors really so uninterested in the B.M.A.'s future? Possibly not; most voluntary bodies tend to be run by a handful of activists, and, furthermore, N.H.S. reorganization seems to have aroused about the same level of enthusiasm among doctors. Nevertheless, this suggests that the $\mathbf{1 5 0}$ odd motions on the agenda of the S.R.M. (Supplement, p. 37) represent the varying opinions of certainly less than $20 \%$ of the membership. This places a heavy responsibility on the representatives, who have to decide the Association's structure for possibly the next 20 years and this in the context of the reorganization of the N.H.S. in 1974.

Several divisions back the Chambers Report in toto, and others support it with a variety of reservations. There is support, too, for the Council's recommendations, which themselves accept some of Sir Paul's ideas but not the backbone of his suggested framework: the exclusion of non-members from B.M.A. activities and the linked proposal to alter drastically the autonomous negotiating structure for N.H.S. doctors. ${ }^{5}$ So the R.B. will not face mutually exclusive alternatives.

Any major constitutional change will require a two thirds majority in the meeting 6 and it is quite on the cards that neither the Chambers Report nor all the Council's recommendations will obtain the necessary majorities. A compromise could well emerge as voting will take place on a succession of key motions. Nevertheless, the end of the day might even see continuation of the status quo. Such an eventuality would raise the question of whether all members should be asked how or whether they would like the B.M.A. to change. The meagre attendance at meetings favours the case for a poll. On a matter of this importance wrong decisions based on the views of a small minority-albeit an informed onewould harm the whole profession.

The dangers inherent in the situation were underlined by a recent editorial on N.H.S. and B.M.A. reorganization in one division's local bulletin.7 "If local response can be taken as a yardstick then participation by doctors in this Division in making representative decisions . . . must give cause for alarm at any decisions made on a national basis." Referring to the professions reliance on the enthusiastic few, it continued: "That such representation, drawn from a shrinking field of interested doctors, must inevitably end in a level of representation that is at best probably adequate but at worst unrepresentative seems to have escaped the non-participating doctor. We have already noted issues on which national decisions, made by available representation, have resulted in bitter recrimination by the non-participating rank and file."

This sombre warning comes from the very guts of the B.M.A.- the division. On 15 November representatives should ask themselves at every vote whether they believe that their yes or no will really improve the B.M.A. where it matters, among the working doctors. The central committees should be prepared to adjust themselves accordingly for they can neither represent nor lead unless they have the confidence of those doctors. The Association has a herculean task in reconciling and representing the many individual and group opinions, but unless individual doctors sense that the B.M.A. is doing this fairly and vigorously it will falter. That is what this S.R.M. is about.

\footnotetext{
1 British Medical fournal Supplement, 1972, 2, 45.

2 British Medical fournal Supplement, 1972, 3, 51.

McVail, John C., The British Medical Association, its Constitution and Government, and Historical Survey. London, B.M.A., 1924.

5 British Medical fournal, 1972, 3, 655 .

6 Memorandum and Articles of Association and By-laws of the British Medical Association. London, B.M.A., 1967, p. 28, para. 40. 7 Medway Bulletin No. 22. Medway and Gravesend B.M.A. Division,
} 